\title{
Estrategias para potenciar el aprendizaje de una lengua extranjera desde el enfoque de la socioformación
}

\author{
Strategies to enhance the learning of a foreign \\ language from the socioformation approach
}

ISMAILA MOUNKORO

Alianza Franco Potosino de San Luis Potosí, México

\begin{abstract}
Resumen
La socioformación se presenta como un enfoque educativo innovador que busca incrementar las habilidades de los alumnos para solucionar los problemas cotidianos de la sociedad. El propósito del presente artículo fue analizar los problemas de aprendizaje de una lengua extranjera (ALE) de acuerdo con los desafíos de la sociedad del conocimiento y determinar los componentes de la socioformación que potencializan dicho aprendizaje. Como metodología de investigación, se realizó un análisis documental sobre los constituyentes de la socioformación y las dificultades del ALE. Los resultados de los análisis determinaron que el aprendizaje basado en problemas (ABP) y por medio de la tecnología como componentes de la socioformación potencializan el aprendizaje de una lengua extranjera. La conclusión principal fue que los componentes de la socioformación son claves para potencializar el ALE, a través del uso particular de los principios del aprendizaje cognitivo, metacognitivo y afectivo combinado con los métodos de la socioformación.
\end{abstract}

Palabras clave: enseñanza-aprendizaje, estrategias, idioma, sociedad del conocimiento, socioformación

\begin{abstract}
The socioformation is presented as an innovative educational approach that asks for increasing the skills of students to resolve the daily problems of society. The purpose of this paper was to analyze the problems of Learning a Foreign Language (known in Spanish as ALE) according to the challenges of the knowledge society and determine the components of socioformation that potentiate such learning. As a research methodology, a documentary analysis was done on the constituents of socioformation and the difficulties
\end{abstract}


of ALE. The results of the analyzes determined that Problem Based Learning (known in Spanish as ABP) and learning through technology as components of socioformation potentialize the Learning of a Foreign Language. The main conclusion was that the components of socioformation are key to potentiate ALE, through the particular use of the principles of cognitive, metacognitive and affective learning combined with the methods of socioformation.

Keywords: teaching-learning, strategies, language, knowledge society, socioformation

\section{Introducción}

$\mathrm{E}$ $n$ esta época de mundialización, el Aprendizaje de una Lengua Extranjera (ALE) se ha convertido en una necesidad indispensable. Como lo indicó World Languages, en 2018, 178 millones de personas hablaban mandarín como segunda lengua; 120 millones, hindú; 70 millones, español; 10 millones, portugués; $70 \mathrm{mi}$ llones, francés; 1 millón japonés; y 28 millones, alemán. Desde el decenio de 1970, los métodos de aprendizaje siempre han sido el centro de la atención en este ámbito, como lo plantean Hosenfeld (1979, 2002), Rubin (1975), Stern (1975) y Wenden $(1991,2002)$ citados por Rahimia y Katala (2012), así como Macaro (2001), Oxford (1990, 1993), Cohen (1990, 1998), Anderson (1991, 2003), O’Malley y Chamot (1990).

¿Qué se puede hacer ante los problemas frente a la necesidad del ALE en la sociedad del conocimiento? Existen dos formas posibles de enfrentar la problemática: continuar con la enseñanza igual que antes o probar algo nuevo. La primera opción debe ser rechazada, ya que no solo será injusta para los estudiantes, sino que también les llevará a una descontextualización de las exigencias del mundo actual (Tobón, Guzmán, Hernández y
Cardona, 2015). La segunda opción es la más apropiada (Harmer, 2015). Para Gómez (2017), la educación necesita nuevos contenidos y metodologías para llevar a cabo de manera pertinente una formación adaptada al contexto actual.

La socioformación como nuevo enfoque consiste no solo en preparar para la sociedad del conocimiento con el uso de la tecnología y con el trabajo colaborativo mediante proyectos formativos, sino también que este nuevo enfoque lleva a resolver problemas en un contexto local, con un panorama global y un sentido crítico (Tobón, 2015). Según Jaleniauskien (2016), dichas competencias son las más importantes para que los estudiantes tengan éxito en sus estudios, en su vida futura y en sus carreras profesionales.

Muchos investigadores destacados como Skinner (1954), Piaget (1993), Vygotski (1987), Ausubel (1968) y Feustein (1991), quienes conformaron diferentes escuelas o tendencias conocidas como conductismo, cognitivismo, constructivismo, histórico-cultural, aprendizaje significativo, interaccionismo, etc., tuvieron una importante influencia en el ALE y demostraron sus límites. Con dichos métodos, los alumnos adquieren básicamente conocimientos gramaticales y sus objetivos de enseñanza son muy generales e imprecisos (Almanza, 2001). Por otra parte, Richards y Renandya 
(2002) comentan que: "la investigación sobre ALE casi siempre produce resultados que están sujetos a interpretación en vez de dar pruebas concluyentes" (p. 11).

Así mismo, Ramírez (2008) demuestra que solo $2 \%$ de las investigaciones sobre procedimientos de enseñanza y aprendizaje de lengua extranjera (ALE), realizado del 2000 al 2010 en México, estuvo concentrado sobre estrategias de aprendizaje. Además, Martínez (2009), después de haber hecho un recorrido de cinco siglos sobre la metodología de enseñanza de un idioma extranjero, termina preguntando: "¿Ahora qué? ¿Existe un método ideal?" (p. 14). Más adelante, el diagnóstico realizado en el 2015 por la Secretaría de Educación Pública de México menciona dos grandes problemas para la enseñanza-aprendizaje de una lengua extranjera: la falta de dominio del idioma por parte de los instructores y las formas de enseñanza en las aulas.

Este artículo analiza los problemas de ALE de acuerdo con los desafíos de la sociedad del conocimiento y determina los constituyentes de la socioformación que potencializan dicho aprendizaje. La sociedad del conocimiento está enfocada en una visión educativa que busca una plena formación de los ciudadanos, atacando los problemas actuales y apoyándose en la cooperación y en la tecnología (Gómez y Tobón, 2017).

\section{Metodología}

Para llevar a cabo este proyecto, como parte de la metodología, se utiliza el análisis documental. Según Arias (2012), el análisis documental es la búsqueda, la organización y el estudio crítico de documentos para responder a unas determinadas preguntas de investigación. Además, para Tobón, Guzmán, Hernández y Cardona (2015), el análisis documental se define como: "un proceso basado en la búsqueda, recuperación, análisis, crítica e interpretación de datos secundarios" (p. 31).

Categorías de análisis. En la tabla 1 , se presenta el análisis de categorías empleadas en el estudio.

\section{Tabla 1}

\section{Categorías de análisis}

\section{Categorías $\quad$ Preguntas o componentes}

Los problemas de ALE de acuerdo con los retos de la sociedad del conocimiento.

Los constituyentes de la socioformación que potencializan el ALE.
¿Cuáles son los problemas de ALE de acuerdo con los retos de la sociedad del conocimiento?

Fuente: elaboración propia. 
Criterios de selección de los documentos. Para elegir los documentos empleados en el análisis, se consideraron los siguientes criterios:

1. Se hizo una investigación de artículos y libros en los portales de Google Académico, WoS, Science Direct, Scielo, Redalyc y Latindex.

2. Se usaron las siguientes palabras clave para realizar la búsqueda: "potencializar, aprendizaje, idioma, sociedad del conocimiento, socioformación y clase invertida".

3. Se seleccionaron artículos de revistas indexadas. En algunos casos,

Tabla 2

Ejemplo de documentos analizados en el estudio se emplearon libros de editoriales reconocidas, centros de investigación o universidades que abordaban categorías del tema.

Documentos analizados. En la tabla 2 , se presentan de forma cuantitativa los documentos elegidos para el estudio en las bases de datos. También se seleccionó una serie de documentaciones complementarias para el análisis y la contextualización, las cuales se presentan en la misma tabla.

\begin{tabular}{ccccc}
\hline Documentos & $\begin{array}{c}\text { Sobre el } \\
\text { tema }\end{array}$ & $\begin{array}{c}\text { De contextualización } \\
\text { o complemento }\end{array}$ & Latinoamericanos & $\begin{array}{c}\text { De otras } \\
\text { regiones }\end{array}$ \\
\hline Artículos teóricos & 14 & 0 & 2 & 12 \\
Artículos empíricos & 26 & 0 & 2 & 24 \\
Libros & 5 & 2 & 2 & 5 \\
Manuales & 0 & 2 & 2 & 0 \\
\hline
\end{tabular}

Fuente: elaboración propia.

\section{Resultados}

En este apartado se muestran los resultados obtenidos según cada categoría.

Categoría 1. Los problemas de ALE de acuerdo con los retos de la sociedad del conocimiento. Con base en la exploración de la literatura, se afirma que aprender un idioma extranjero es un proceso complejo y, por ello, existen numerosos factores que afectan el ALE. Dichos factores se pueden asociar en tres grandes grupos: ambientales, relacionados con el instructor e individuales (Díez, 2010). Entre los factores ambientales que dificultan el ALE, se destacan las condiciones del aprendizaje, el uso del material adecuado, así como la cantidad de alumnos en el 
salón (Henter, 2014). Otros factores ambientales por tomar en cuenta son el grado de semejanza que puede haber entre el idioma materno y el idioma por aprender, cuanta más semejanza haya entre la lengua madre y la lengua por aprender, más fácil será la adquisición del nuevo idioma (Valero y Jiménez, 2015). Los factores ambientales dan más importancia a lo adquirido y no a lo innato (Moreno, 2007).

Además de los factores ambientales, hay otro muy importante relacionado con el docente, si es nativo o es un segundo idioma adquirido el que enseña (Valero y Jiménez, 2015). Según Medgyes (2001), el docente nativo puede efectuar un excelente trabajo gracias a su amplio e implícito conocimiento de la lengua por enseñar; además, es más innovador, usa más materiales, tolera más los errores y comparte más información cultural. Sin embargo, Medgyes mismo menciona más adelante que el docente no nativo tiene una experiencia por el hecho de haber aprendido esa lengua y así puede anticipar soluciones a los problemas que los alumnos podrían enfrentar y tener una mayor empatía. Otros factores que se pueden relacionar con el docente son: la especialización, la constante capacitación y la motivación por la docencia (Díez, 2010).

Varios autores han expresado factores individuales que afectan el ALE. De acuerdo con Richards y Renandya (2002), el éxito en el ALE depende mucho de la autoestima. Se ha evidenciado que la edad es un factor muy determinante, así se considera la niñez como la etapa más adecuada para aprender un idioma extranjero, ya que el cerebro presenta una flexibilidad que alienta hacia nuevos aprendizajes (Díez, 2010; Mayo y Lecumberri, 2003; Mercau, 2009; Roca y Manchón, 2006, citados por Valero y Jiménez, 2015). Para Valero y Jiménez (2015), el periodo más adecuado para aprender una lengua es cuando el cerebro y el sistema cognitivo tienen capacidad de ejecutar operaciones formales. Sin embargo, Mayberry, Lock y Kazmi (2002) mencionan que la edad no es un factor determinante en el ALE.

El inadecuado diseño curricular, la cantidad elevada de alumnos por salón, la falta de capacitación permanente de los docentes, así como la carencia de materiales adecuados o metas poco realistas son otros factores negativos señalados por DiFino y Lombardino (2004). La poca capacidad de memoria, el déficit de interés o de atención, además de una lengua materna adquirida inadecuadamente son algunas otras dificultades (Valero y Jiménez, 2015; Mayberry, Lock y Kazmi, 2002). Otros elementos por tomar en cuenta son: las capacidades afectivas, la falta de motivación y la actitud negativa (Henter, 2014). Además, la ansiedad que se presenta de manera frecuente o escasez de metodología de aprendizaje (Valero y Jiménez, 2015; Arnold, 2000).

Categoría 2. Los constituyentes de la socioformación que potencializan el ALE. Con el objetivo de poner al día a los estudiantes, mejorar su capacidad y enriquecer la calidad general del proceso de enseñanza-aprendizaje, un número considerable de innovaciones educativas han surgido. La socioformación se define, según Tobón (2015), como un enfoque educativo innovador que busca incrementar las habilidades de los alumnos para solucionar los problemas 
cotidianos de la sociedad. Este enfoque es la base de varias de dichas innovaciones. Una de las innovaciones propuestas por la socioformación son los proyectos formativos que buscan la preparación de las personas para que puedan resolver problemas cotidianos enfocándose sobre el trabajo colaborativo (Tobón, Cardona, Vélez y López, 2015).

Según Jonassen (2011), el ABP es la innovación curricular más significativa en la historia de la educación. Esta innovación y el trabajo colaborativo son partes fundamentales de los proyectos formativos. Para Doghonadze y Gorgiladze (2008), el ABP es una forma de enseñanza enfocada en el principio de usar problemas como la base de la asimilación e integración de los nuevos conocimientos. Estos autores agregan que es un aprendizaje centrado sobre los alumnos y son dueños de sus aprendizajes bajo la supervisión de un profesor; el aprendizaje se produce en pequeños grupos y favorece la empatía, así como la motivación en los alumnos, elementos indispensables para alcanzar un aprendizaje significativo.

Por otra parte, Morales y Landa (2004) señalan que el ABP está enfocado en la cooperación y que la enseñanza es más eficiente cuando hay intercambio de ideas y cuando todos los actores colaboran para resolver un problema. En lugar de una enseñanza centrada en el instructor, el aprendizaje basado en problemas (ABP) utiliza un enfoque de aprendizaje contextualizado y dirigido por los alumnos preparándolos para los desafíos del mundo real (Doghonadze y Gorgiladze, 2008).

Jaleniauskien (2016) puntualiza que este método es un aprendizaje vivo y compromete a los aprendices a la discusión, al debate y a la controversia, donde la curiosidad intelectual es la esencia del aprendizaje del estudiante. Por lo tanto, el ALE requiere su revitalización con la introducción de los proyectos formativos a través del ABP. Los estudiantes aprenden a pensar críticamente, a seleccionar material significativo, a interactuar entre ellos en el proceso de investigación sobre soluciones a los problemas de realidad cotidiana, abandonando los viejos libros de texto y desarrollando un enfoque más profundo en el aprendizaje (Jaleniauskien, 2016; Savin-Baden, 2000).

El otro constituyente de la socioformación que potencializa el ALE es la enseñanza y el aprendizaje por medio de las tecnologías. Considerado según Becta (2010) como la clave del progreso, el uso de la tecnología contribuyó a desarrollar varias iniciativas educativas. Una de las formas prácticas de reforzar la enseñanza de una lengua extranjera es consolidar la utilización de las tecnologías en la educación. Su uso en el ALE ayuda a intensificar y a personalizar el aprendizaje; además, promueve el interés en los estudiantes (Bilyalova, 2017). Gracias a estas prácticas se puede evitar la evaluación subjetiva e, igualmente, las tecnologías contribuyen a superar la barrera psicológica de los estudiantes (Hubackova y Ruzickova, 2011).

Así mismo, Rahimi y Shahab (2014) consideran que las tecnologías influyen en el aprendizaje del vocabulario, promueven la interacción alumno-alumno y aumentan la capacidad comunicativa. Estos son factores que incitan el aprendizaje de idiomas y también incluyen una variedad de aplicaciones que favorecen un aprendizaje de manera más eficiente, 
posibilitando a los alumnos la búsqueda de más palabras y frases, encontrar sinónimos y antónimos, e inclusive ayudándoles en la pronunciación.

Hubackova y Ruzickova (2011) aseguran que el uso de la tecnología produce un cambio de mentalidad en los estudiantes respecto a las tareas. Este uso logra que dejen de ver las tareas como algo rutinario para convertirlas en un trabajo agradable. También Buga, CăpeneaĞă, Chirasnel y Popa (2014) señalan que el uso de la tecnología facilita y agiliza el trabajo de los alumnos.

Un constituyente fundamental de la socioformación es el proyecto ético de vida. Este consiste en analizar el porqué de su vida, es decir, sus metas, reforzando las relaciones sociales y respetando la naturaleza. Se basa en aspectos como la realización personal, las competencias, tener metas y laboriosidad (Tobón, 2015). Todos esos elementos son parte fundamental del fortalecimiento del aprendizaje de una lengua extranjera, puesto que la primera meta para aprenderla es la realización personal, viajar, trabajar, descubrir. Una lengua extranjera no se puede aprender sin tener ciertas competencias y habilidades, además de tener mucha laboriosidad.

\section{Conclusión}

Con el análisis documental llevado a cabo, una primera conclusión es que tanto las dificultades como las estrategias de aprendizaje han existido y han evolucionado en función de la sociedad. En la sociedad del conocimiento, se observa que los factores afectivos, socioeconómicos de los alumnos, así como la falta de contextualización del aprendizaje, el desamor y la ausencia de compromisos en la docencia han sido poco investigados.

Una segunda conclusión es que la socioformación se presenta, comparada con otros enfoques, como el más adecuado para aprender un idioma. En la socioformación, se encuentran todas las herramientas indispensables para aprender una lengua en la sociedad del conocimiento. Según Tobón (2015), es el único enfoque que se centra en la formación integral. El uso de las tecnologías, el trabajo colaborativo y el ABP responden de manera idónea a los retos de aprendizaje en general y de los idiomas en particular.

Morales y Landa (2004) mencionan que el trabajo colaborativo es más eficiente en el aprendizaje de un idioma. Por una parte, Jonassen (2011) dice que el $\mathrm{ABP}$ es la innovación más significativa en la educación. Por otra, Bilyalova (2016) señala que el uso de la tecnología se presenta como un instrumento vital en la educación en la sociedad de conocimiento. Para este mismo autor, el uso de la tecnología es, sin duda, una de las maneras más prácticas de reforzar el ALE.

Otra estrategia similar a la de la socioformación es "La clase invertida" que debe mucho a Salman Kahn y a dos profesores de Química en una preparatoria de Colorado en Estados Unidos. Fue un gran éxito en América del Norte desde los años 2000 (Dufour, 2014). Para Bennett et al. (2011), "la clase invertida" es un método que se enfoca en el uso de la tecnología y el trabajo colaborativo. Permite pasar de un método centrado sobre el maestro al alumno y así tener clases más agradables y alumnos más motivados, 
más activos, autónomos y cooperativos; además, les abre al mundo exterior por medio de los proyectos y así tener mejores resultados.

\section{Discusión}

De acuerdo con Chilingaryan y Zvereva (2016), la esencia de este método es la autonomía en las actividades cognitivas de bajo nivel, priorizando el trabajo colaborativo y las tareas de aprendizaje cognitivo de alto nivel en el aula. Sin duda, la socioformación ofrece bastantes herramientas para potencializar el ALE; no obstante, siguen vigentes estas preguntas: ¿Qué estrategias proponer para reforzar los factores personales, si se sabe que hay alumnos que, aun estando en la sociedad del conocimiento, rechazan el uso de la tecnología o el trabajo colaborativo? ¿Qué hacer con esas comunidades rurales que no tienen electricidad y mucho menos tecnologías? ¿Cómo lograr una enseñanza más eficiente con grupos heterogéneos?

El mundo va evolucionando y los métodos se deben adaptar a este cambio, para lo cual se necesita más investigación que permita determinar el papel preciso de las formas de aprendizaje del idioma. Los profesores deberían tener más conciencia y estar más comprometidos para ayudar, trabajando sobre la singularidad de cada estudiante. Es imprescindible determinar el rol de todos los actores en el proceso de la enseñanza y aprendizaje. Finalmente, es indispensable un estudio más profundo sobre la adquisición de cada competencia, en particular, la escrita y la oral.

\section{Bibliografía}

Almanza, G. (2001). Alternativa Metodológica para una Enseñanza Participativa de la Lengua Inglesa con Fines Específicos en la Facultad de Contabilidad y Finanzas (Tesis de Maestría en Ciencias de la Educación Superior). Universidad de Ciego de Ávila, Cuba.

Arias, F. (2012). El Proyecto de Investigación. Introducción a la metodología científica. Caracas: Episteme. Arnold, J. (2006). Los factores afectivos en el aprendizaje del español como lengua extranjera. Recuperado de goo.gl/zRdcqU

Ausubel, D. P. (1963). The psychology of meaningful verbal learning. Nueva York: Grune and Stratton.

Bennett, B.E., Spencer, D., Bergmann, J., Cockrum, T., Musallam, R., Sams, A., Fisch, K. y Overmyer, J. (2011). The Flipped Class Manifest. Recuperado de goo.gl/njzZNA

Bilyalova, A. (2017). ICT in Teaching a Foreign Language in High School. Procedia - Social and Behavioral Sciences, 237, pp. 175-181. Recuperado de goo.gl/N7EW3F

Buga, R., CăpeneaĞă, L., Chirasnel, C. y Popa, A. (2014). Facebook in foreign language teaching - a tool to improve communication competences. Procedia - Social and Behavioral Sciences, 128, pp. 93-98. Recuperado de goo.gl/ndfLrj

Cohen, A. D. (1996). Second language learning and use strategies: Clarifying the issues. Recuperado de https://www.researchgate.net/publication/237361003_Second_language_learning_and_use_strategies_Clarifying_the_issues 
DiFino, S. M. y Lombardino, L. J. (2004). Language learning disabilities: the ultimate foreign language challenge. Foreign Language Annals, 37(3), pp. 390-400. Recuperado de goo.gl/3zgqiS

Doghonadze, N. y Gorgiladze, G. (2008). Problem solving in teaching foreign languages to students of pedagogical departments. IBSU Scientific Journal, 2(1), pp. 101-114. Recuperado de goo.gl/Sz3dF9

Dufour, H. (2014). La classe inversée. TECHNOLOGIE, 193, pp. 44-47 Recuperado de goo.gl/Dg4rnu

Dulzaides, M. E. y Molina, A. M. (2004). Análisis documental y de información: dos componentes de un mismo proceso. Acimed, 12(2), pp. 1-5. Recuperado de https://goo.gl/b9o50C

Gómez, J. y Tobon, S. (2017). Análisis documental de los proyectos formativos en Educación Básica. Metodología, experiencias y beneficios. Revista Atlante: Cuadernos de Educación y Desarrollo. Recuperado de goo.gl/ef7tDK

Harmer, J. (2015). The Practice of English Language Teaching. Inglaterra: Pearson Education Limited.

Henter, R. (2014). Affective factors involved in learning a foreign language. Procedia - Social and Behavioral Sciences, 127, pp. 373-378. Recuperado de goo.gl/5uHGk7

Hubackova, S. y Ruzickova, M. (2011). Experience in foreign language teaching with ICT support. Procedia Computer Science, 3, pp. 243-247. Recuperado de goo.gl/dp1rP5

Jaleniauskien, E. (2016). Revitalizing Foreign Language Learning in Higher Education Using a PBL Curriculum. GlobELT, 14-17, pp. 265-275. Recuperado de goo.gl/ $\mathrm{XXuDk} 4$
Jonassen, D. H. (2011). Learning to solve problems: A handbook for designing problem-solving learning environments. Nueva York y Londres: Routledge. Recuperado de goo.gl/EvvGUg

Mayberry, R. y Lock, E. (2003). Age constraints on first versus second language acquisition:Evidence for linguistic plasticity and epigenesis. Brain and Language, 87, pp. 369394. Recuperado de goo.g//UHpU1Q Medgyes, P. (2001). When the teacher is a non-native speaker. Teaching English as a second or foreign language, 3, pp. 429-442. Recuperado de goo.gl/SZfbmV

Moral M. E., Villalustre, R. (2014). Oportunidades de las TIC para la innovación educativa en las escuelas rurales de Asturias. Aula abierta, 42, pp. 61-67. Recuperado de goo.gl/jvTzr

Morales, B. P. y Landa, F. V. (2004). Aprendizaje basado en problemas. Theoria, 13, pp. 145-157. Recuperado de goo.gl/SQxk8B

Moreno, R. (2007). Adquisición de segundas lenguas y Sociolingüística. Revista de Educación, 343, pp. 5570. Recuperado de goo.gl/b8nBAF

O'Malley, J. M., Chamot, A.U. y Küpper, L. (1989). Listening comprehension strategies in second language acquisition. Applied Linguistics, 10, pp. 418-37. Recuperado de goo.gl/c7wQYh

Oxford, R. L. (1990). Language learning strategies: What every teacher should know. Nueva York: Newbury House Publishers. Recuperado de goo.gl/iATa3n

Rahimi, M. y Shahab, M. (2014). International Conference on Current Trends in ELT The Impact of Mobile 
Dictionary Use on Language Learning. Procedia - Social and Behavioral Sciences, 98, pp. 1469-1474. Recuperado de goo.gl/E31gDQ

Ramírez, J. L. (2008). Las Investigaciones sobre las enseñanza y el aprendizaje de lenguas extranjeras en México. México: Plaza y Valdés. Recuperado de goo.gl/YAueZ

Richards, J. C. y Renandya, W. A. (Eds). (2002). Methodology in language teaching: An anthology of current practice. Cambridge: Cambridge University Press. Recuperado de goo.gl/kKWG2b

Savin-Baden, M. (2000). Problembased learning in higher education: Untold stories. Great Britain: SRHE and Open University Press. Recuperado de: goo.gl/4GTc8v

SEP. (2008). La enseñanza de idiomas en México. Diagnóstico, avances y desafíos. Recuperado de goo.gl/dgkTzp

Tobón, S., Cardona, S., Vélez, J. y López, J. (2015, enero-diciembre). Proyectos formativos y desarrollo del talento humano para la sociedad del conocimiento. Acción pedagógica, (24), pp. 20-31. Recuperado de https://goo.gl/8bakfC

Tobón, S., González, L., Salvado, J. y Manuel, J. (2015). La Socioformación: Un Estudio Conceptual. Paradigma, XXXVI, 23. Recuperado de goo.gl/YVLKUh

Tobón, S., Guzmán, C. E., Hernández, J. S. y Cardona, S. (2015). Sociedad del Conocimiento: Estudio documental desde una perspectiva humanista y compleja. Revista Paradigma, 36(2), pp. 7-36. Recuperado de goo.gl/HQwhBX

Valero, N. y Jiménez, G. (2015). Estudio exploratorio sobre dificultades en el aprendizaje de una segunda lengua: la opinión del profesorado. Espiral. Cuadernos del Profesorado, 8(16), pp. 3-12. Recuperado de goo.gl/e8Urk2

Vygotsky, L. (1978). Mind in society. Cambridge, MA: Harvard University Press. 